\title{
Striking the balance on AIDS
}

SIR-The "Sombre view of AIDS" proposed by Malcolm Rees (Nature326, 343$345 ; 1987)$ is based on the suggestion of a 15-year mean incubation for the human immunodeficiency virus (HIV) transferred by blood transfusion and a consequentially high estimate of future incidence. He uses Peterman's data (given in Rees's Table 1). His mode of analysis is perhaps too indirect. At all events the simple 'actuarial' approach leads to a different conclusion.

Write $p$, for the chance of AIDS (acquired immune deficiency syndrome) diagnosis in the $s$-th year after infection. The ratio $r_{\mathrm{s}}=p_{\mathrm{s}+1} / p_{\mathrm{s}}$ may be directly estimated from the diagonal totals in Table 1 relating to the same set of annual cohorts of infecteds. The estimates (for the five values $s=1 . .5$ whose measurement the data permits) are:

$$
5.00,1.77,1.59,0.84,1.18
$$

This is not consonant with a gaussian distribution with $\mu=15, \sigma=5$ which gives values:

\section{$1.73,1.67,1.63,1.56,1.47$}

The observed ratios suggest the probabilities rise rapidly to a peak around five years. Rees's suggestion of a log-normal graduation could give rise to ratios with this characteristic. So equally would graduation using other distributions like the gamma (which arises for example as a special case of the analogous distributions advocated by Julian Peto in carcinogenesis using staged models). Whatever form is used for graduation the salient feature is the same: a mean incubation of about five years rather than fifteen. This leads to an order of magnitude reduction in Rees's "sombre" projections.

\section{Department of Computer Science and Statistics, \\ Queen Mary College, \\ University of London, \\ Mile End Road, \\ London E1 4NS, UK}

SIR-Your report (Nature 324, 611; 1986) on AIDS in Africa highlights "the sorry state of affairs that exists between French and US AIDS researchers" and a possible "African origin of AIDS". It seems to us that such perceived problems are unduly empha-sized by an overzealous press, further complicating attempts to conduct important research. We have, for example, maintained an international agreement for a trilateral study on AIDSrelated viruses with both French and US investigators. This arrangement has been focused on common goals, based on mutual respect, and has been free of problems related to nationalism.

Perhaps a more serious concern for Africa is the need for careful and objective evaluations of experimental results that could have major implications for public health policies. Some of the first seroepidemiological studies conducted in Africa reported large proportions of ELISA (enzyme-linked immunosorbent assay) test results that were found to be false positives. This experience may have helped to create a climate of reluctance to accept the sub-sequent results that accurately revealed the extent of the epidemic in Central and East Africa.

In West Africa we now have a situation where a significant proportion of people have been exposed to a different class of retrovirus that is highly related to the primate retrovirus, STLV-3, and variously designated HTLV-IV, LAV-2 and HIV-2. Premature conclusions that this new class of retrovirus is as pathogenic as HIV-1 could result in unsound policy decisions in various West African countries. It is particularly important that appropriately controlled population-based studies be conducted to address the possible link between infection with this new class of retrovirus and AIDS or any other pathology. Such studies should be conducted as soon as possible and should incorporate highly specific assays that allow one to distinguish between HIV-1 and HIV-2. Only then can a sensible and sound health policy response be formulated.

S. Mboup, D. Ricard, C. Boye, A. Gaye, J.L. SAnKale Laboratorie de Bacteriologie-Virologie, Faculté de Medécine et de

Pharmacie de Dakar,

Dakar-Fann, Senegal

Bureau des MST,

I. NDOYE

Ministére de la Sante,

Dakar, Senegal

BP 8175, Ouadougou, Burkina-Faso

BP 08, Foumban, Cameroun

BP 217 bis, Conakry, Guinée

M. Rajaonarivoy

BP 460 Antanarivo, Madagascar

\section{Singapore maligned}

SIR-A letter under the headline "Dissent disallowed in Singapore" (Nature 324, 298 ; 1986) contained factual inaccuracies and appears to be an unprovoked attempt to discredit Singapore before the international scientific community. The Fourth Federation of Asian and Oceanic Biochemists Congress that took place in Singapore was the most recent in a series of triennial regional meetings organized by biochemists of the Asia-Pacific region. As such, its planning and eventual success as a scientific meeting did not depend on aggressively seeking the participation of the international scientific community. That this meeting attracted about $700 \mathrm{reg}$ istrants from 33 countries is perhaps the most telling evidence that scientists are not facile targets for alarmists.

The congress was organized independently of the planning of the Institute of Molecular and Cell Biology in Singapore. No attempt was made to recruit scientific staff for this institute at the meeting.

The statement that "the voice of dissent in Singapore... is to be disallowed at all cost" is false and invidious as is the grotesque suggestion that scientists elsewhere should use their influence to rescue the people of Singapore from their unpleasant fate.

163, Sixth Avenue,

EDWARD JACOB

Singapore 1027

\section{Papal IVF poser}

SIR-Your leading article on the Vatican's pronouncements on the ethics of in vitro fertilization (Nature 326, 229-230; 1987) leads me to wonder if the unfortunate couples who have been determined to be mutually infertile have the right in God's eyes to engage in intercourse? After all, it could be considered that 'science' has determined that there is no procreative future in such an act, and so the kind permission to engage in "natural acts which are intrinsically directed towards procreation" must most regrettably be withheld. If only scientists were unable to diagnose sterility perhaps these couples would at least have the right to keep on trying.

Nostra maxima culpa!

39 , rue St Jean,

T.B. GREENLAND

69005 Lyon, France

\section{Three degrees}

SIR-In K.S. Jayaraman's article on "Superconductivity" (Nature 326, 237; 1987), there are some factual errors.

(1) There are several teams working on superconductivity in India and the team that reported, following the work of Chu et al., the highest superconducting onset and zero-resistance tempeature for the first time is solely from the Tata Institute of Fundamental Research (TIFR). No scientist from the Bhabha Atomic Research Centre (BARC) is involved.

(2) The zero resistance state is reached at $87 \mathrm{~K}$ and not at $90 \mathrm{~K}$.

(3) This is not the same team as the one that a month ago reported superconductivity in a lanthanum oxide system (which is of course a BARC-TIFR team).

E.V. SAMPATHKUMARAN

Solid State Physics Group,

Tata Institute of Fundamental Research, Bombay 400 005, India 